\title{
Arthroscopic Superior Capsule Reconstruction Eliminates Pseudoparalysis in Patients with Irreparable Rotator Cuff Tears
}

\author{
Teruhisa Mihata, MD, PhD1, Thay Q. Lee, PhD², AKIHIKO HASEGAWA, MD, PhD³, Takeshi Kawakami, MD, PhD4, \\ Kunimoto Fukunishi, MD ${ }^{5}$, Yukitaka Fujisawa, MD, PhD $^{6}$, Yasuo Itami, MD7 , Mutsumi Ohue, MD ${ }^{8}$, Masashi Neo, MD, PhD ${ }^{9}$ \\ ${ }^{1}$ Department of Orthopaedic Surgery, Osaka Medical College, Takstsuki, Osaka, Japan, ${ }^{2}$ VA Long Beach HCS and University of \\ CA, Long Beach, CA, USA, ${ }^{3}$ Osaka Medical College, Takatsuki, Japan, ${ }^{4}$ First Towakai hospital, Takatsuki, Japan, ${ }^{5}$ Shimizu \\ hospital, Kyoto, Japan, ${ }^{6}$ Yaenosato hospital, Higashiosaka, Japan, ${ }^{7}$ Department of Orthopaedic Surgery, Osaka Medical college, \\ Takatsuki, Japan, ${ }^{8}$ Katsuragi Hospital, Kishiwada, Japan, ${ }^{9}$ Department of Orthopaedic Surgery, Osaka Medical College, \\ Takatsuki, Japan.
}

Objectives: We have developed the superior capsule reconstruction (SCR) technique for surgical treatment of irreparable rotator cuff tears. In these patients, SCR restores shoulder stability and muscle balance, consequently improving shoulder function-particularly deltoid muscle function-and relieving pain. In this study, we evaluated whether arthroscopic SCR reversed preoperative pseudoparalysis in patients with irreparable rotator cuff tears. Methods: A series of 100 consecutive patients with irreparable rotator cuff tears that had failed conservative treatment underwent arthroscopic SCR using fascia lata autografts; 7 patients with deltoid weakness due to cervical or axillary nerve palsy and 3 patients with severe shoulder stiffness (passive shoulder elevation, less than $90^{\circ}$ ) before surgery were excluded from the study population. The remaining 90 patients were allocated into 3 groups according to their preoperative active shoulder elevation: (1) no pseudoparalysis: more than $90^{\circ}$ of active shoulder elevation (48 patients; mean age, 66.3 years; mean tear size in anterioposterior direction, $3.5 \mathrm{~cm}$ ); (2) moderate pseudoparalysis: no shoulder stiffness, less than $90^{\circ}$ of active shoulder elevation, patients maintained more than $90^{\circ}$ elevation once the shoulder was elevated passively (27 patients; mean age, 68.1 years, mean tear size, $3.5 \mathrm{~cm}$ ); and (3) severe pseudoparalysis: no shoulder stiffness, less than $90^{\circ}$ of active shoulder elevation, patients had a positive drop-arm sign (15 patients; mean age, 62.3 years, mean tear size, $4.9 \mathrm{~cm}$ ). Physical examination, radiography, and magnetic resonance imaging were performed before surgery; at 3, 6, and 12 months after surgery; and yearly thereafter. The American Shoulder and Elbow Surgeons (ASES) score, active shoulder range of motion, acromiohumeral distance, and healing rate were compared between patients with and without pseudoparalysis as well as between before surgery and at the final follow-up (mean, 48 months; range, 24 to 88 months) by using the $t$ and chi-square tests. A significant difference was defined as $P<0.05$.

Results: ASES score, active elevation, active external rotation, and acromiohumeral distance increased significantly after arthroscopic SCR in patients with no pseudoparalysis, moderate pseudoparalysis, or severe pseudoparalysis. The graft healing rate was $96 \%$ (43 of 45 ) in patients with no pseudoparalysis, $96 \%$ (26 of 27 ) in those with moderate pseudoparalysis, and $87 \%$ (13 of 15 ) in the severe pseudoparalysis group. Postoperative ASES score, active elevation, active external rotation, acromiohumeral distance, and healing rate did not differ among the 3 patient groups. Pseudoparalysis was reversed in 96\% (26 of 27) of patients with moderate pseudoparalysis and in 93\% (14 of 15) patients with severe pseudoparalysis. Patients with residual moderate or severe pseudoparalysis had graft tears postoperatively.

Conclusion: Arthroscopic SCR improved shoulder function and achieved superior stability in patients with previously irreparable rotator cuff tears both with and without pseudoparalysis. Providing that the graft did not tear postoperatively, arthroscopic SCR reversed preoperative pseudoparalysis. The graft healing rate after arthroscopic SCR did not differ between patients with and without pseudoparalysis.

The Orthopaedic Journal of Sports Medicine, 5(3)(suppl 3)

DOI: $10.1177 / 2325967117$ S00119

CThe Author(s) 2017

This open-access article is published and distributed under the Creative Commons Attribution - NonCommercial - No Derivatives License (http://creativecommons.org/licenses/by-nc-nd/3.0/), which permits the noncommercial use, distribution, and reproduction of the article in any medium, provided the original author and source are credited. You may not alter, transform, or build upon this article without the permission of the Author(s). For reprints and permission queries, please visit SAGE's Web site at http://www.sagepub.com/journalsPermissions.nav. 